\title{
Foamy viruses bubble on
}

\section{Robin A. Weiss}

FOR many years we have assumed that all retroviruses replicate by a uniform major strategy. Given that, a paper in last week's Science ${ }^{1}$ provides pause for thought - it shows that the spumavirus human foamy virus (HFV) differs from oncoviruses (such as mouse leukaemia virus, MLV) and lentiviruses (such as HIV) in carrying fulllength, double-stranded DNA genomes in a proportion of virus particles. Furthermore, $\mathrm{HFV}$ reverse transcriptase and other Pol proteins are translated from a spliced messenger RNA, and lack Gag sequences ${ }^{1,2}$. These findings are unexpected and provocative; HFV resembles hepatitis $B$ virus, which has a DNA genome, in these respects as much as it does retroviruses. Moreover, other reports suggest that HFV is not so human after all ${ }^{3}$, but may serve as a useful gene vector to non-proliferating cells ${ }^{4}$.

Retroviruses have an RNA genome but insert a DNA copy of it into the host chromosome following reverse transcription. The genomes of the three retrovirus subfamilies (onco-, lenti- and spumaviruses) each carry at least three genes, gag-pol-env, running $5^{\prime}$ to $3^{\prime}$, which are packaged in virions as an RNA dimer, with cellular transfer RNA acting as a primer for reverse transcription. It was previously thought that DNA synthesis is only initiated in the cytoplasm of the newly infected cell. Yu et al. ${ }^{1}$ show that full-length, DNA proviral genomes, some 12 kilobases long, are present in 10-15 per cent of the particles. By contrast, analysis with the polymerase chain reaction indicates that about 1 in 100,000 virions of onco- or lentiviruses have associated full-length DNA. However, the HFV virion DNA does not appear to be infectious and inhibitors of reverse transcriptase indicate that the enzyme is still required at an early stage of infection.

Although foamy viruses have essentially the same main gene organization as other retroviruses (plus accessory bel genes which lie 3' to env), the finding that pol is translated from its own spliced mRNA, independently of gag, is also quite novel. Other retroviruses synthesize Pol products as part of a Gag-Pol fusion protein, translated either by ribosomal -1 frameshift towards the end of Gag, or by use of a suppressor tRNA. Thus Pol is incorporated into virions via Gag domains in the precursor protein before cleavage by viral protease. As Yu et al. point out, their results pose the question of how Pol is assembled into viral particles.

When I last wrote about foamy viruses in these columns ${ }^{5}$ I called them viruses in search of a disease. Although they are the most cytopathic, syncytium-inducing retroviruses for many cell types in vitro, disease is seldom seen in the many species of pri- mate that they naturally infect. However, mice transgenic for HFV suffer neurodegeneration resembling amyotrophic lateral sclerosis $^{6}$, and virus expression was evident in the brain of an orang-utan which died with similar acute neurological symptoms ${ }^{7}$. But HFV has not been found in human amyotrophic lateral sclerosis ${ }^{8}$, and the various claims that HFV is associated with de Quervain $^{5}$ or Graves ${ }^{9}$ diseases of the thyroid has now been questioned ${ }^{10}$. Indeed there is no longer definitive evidence of human infection, other than in a handful of

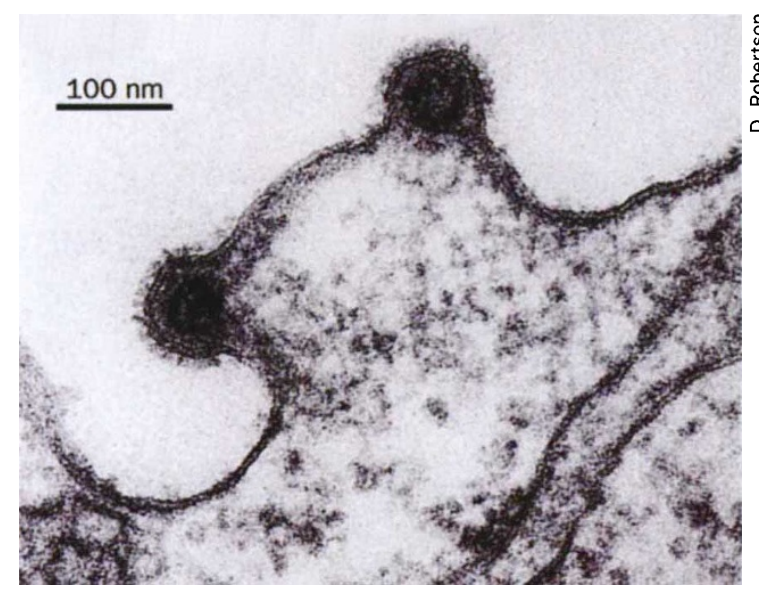

Budding particles of foamy retrovirus, imaged by electron microscopy; note the prominent glycoprotein spikes and the preformed spherical cores. As seen through the light microscope, cell cultures infected with the virus look foamy, hence the name - spumaviruses - of the subfamily.

persons with zoonosis from contact with primates $^{3}$. HFV was the first 'human' retrovirus to be described ${ }^{11}$, yet it is now apparent that the HFV genome sequence is indistinguishable from that of one of the chimpanzee isolates ${ }^{12}$. Following an international workshop on foamy viruses in 1994, at which all laboratories working on foamy virus were represented, exchange and analysis of samples did not uphold evidence for human foamy virus in patients with autoimmune disease.

Given that the great apes so frequently harbour foamy viruses ${ }^{7,12,13}$, it is odd that Homo sapiens has escaped endemic infection. The few persons who have become infected with simian foamy virus (SFV) remain well, have a 'full house' of antibody responses analysed by Western blot, and have viral genomes easily detected in blood by the polymerase chain reaction ${ }^{3}$. These observations have two implications: that reports of widespread human infection are tenuous at most; but that humans can nonetheless be infected by SFV, and in all probability would become infected following xenotransplantation of primate tissue. Thus the patient with AIDS who has received a baboon bone-marrow transplant, and any future organ transplants from primates, should be carefully monitored for foamy virus transfer as well as other viruses.

Parodying Koch's postulates for modern virology, one might propose that if a virus fails as a pathogen, promote it as a vector in gene therapy. This is precisely what has been done for HFV. Schmidt and Rethwilm ${ }^{14}$ deleted the bel2-3 region, while Russell and Miller ${ }^{4}$ substituted marker genes in place of env and bell. With its very broad host range and tropism for cell types ${ }^{4}, \mathrm{HFV}$ may prove to be quite a useful vector.

Russell and Miller further noted that their foamy virus vectors were able to infect cells in the stationary phase of the cell cycle, and might thus be used to deliver genes to non-proliferating targets such as neurons. On the face of it, this finding appears to run counter to our recent report ${ }^{15}$ that HFV replication is cell-cycle dependent. These apparently contradictory results stem largely from a different emphasis in interpretation, however - in both cases, the efficiency of infection of stationary cells was approximately 5-10 per cent that of proliferating cells. Thus HFV infection is less stringently dependent on mitosis than MLV, but is more dependent than HIV. An attractive explanation for HFV infection of non-proliferating cells would be that it occurs through the DNA genomes found in 10-15 per cent of virions (though Yu et al. ${ }^{1}$ thought that the DNA was not infectious). Perhaps Russell and Miller should check whether their vectors can infect stationary cells in the presence of inhibitors of reverse transcriptase.

Robin A. Weiss is in the Chester Beatty Laboratories, Institute of Cancer Research, 237 Fulham Road, London SW3 6JB, UK.

1. Yu, S. F., Baldwin, D. N., Gwynn, S. R., Yendapilli, S. \& Linial, M. L. Science 271, 1579-1582 (1996).

2. Enssle, J., Jordan, I., Mauer, B. \& Rethwilm, A. Proc. natn. Acad. Sci. U.S.A. (in the press).

3. Schweizer, M. et al. AIDS Res. hum. Retrovir. 11, 161-170 (1995).

4. Russell, D. W. \& Miller, A. D. J. Virol, 70, 217-222 (1996).

5. Weiss, R. A. Nature 333, 497-498 (1988).

6. Bothe, K., Aguzzi, A., Lassman, H., Rethwilm, A. \& Horak, I. Science 253, 555-557 (1991).

7. McClure, M. O. et al. J. Virol. 68, 7124-7130 (1994).

8. Rösener, M., Kranz, M., Hahn, H., Heeney, J. \& Rethwilm, A. J. med. Virol. (in the press).

9. Lagaye, S. et al. Proc. natn. Acad. Sci. U.S.A. 89, 10070-10074 (1992).

10. Schweizer, M., Turek, R., Reinhardt, M. \& NeumannHaefelin, D. AIDS Res. hum. Retrovir. 10, 601-605 (1994).

11. Achong, B. G., Mansell, P. W. A., Epstein, M. A. \& Clifford, P. J. J. gen. Virol. 40, 175-181 (1971).

12. Herchenröder, O. et al. Virology 201, 187-199 (1994).

13. Bieniasz, P. D. et al. Virology 207, 217-228 (1995).

14. Schmidt, M. \& Rethwilm, A. Virology 210, 167-178 (1995).

15. Bieniasz, P. D., Weiss, R. A. \& McClure, M. O. J. Virol. 69, 7295-7299 (1995). 